\title{
ON THE STRUCTURE OF EQUILIBRIUM PHASE TRANSITIONS WITHIN THE GRADIENT THEORY OF FLUIDS*
}

\author{
BY \\ MORTON E. GURTIN (Carnegie-Mellon University) \\ AND \\ HIROSHI MATANO ( Hiroshima University, Japan)
}

1. Introduction. In the van der Waals/Cahn-Hilliard theory ([20], [19], [4]) of phase transitions, the density distribution of a two-phase fluid is based on a freeenergy that depends not only on the density $u(x)$, but also on the density gradient $\nabla u(x)$. We shall consider this theory, but only with a relatively simple dependence on $\nabla u$, and only as restricted to isothermal situations; precisely, we shall allow for a free-energy (per unit volume) of the form

$$
W(u(x))+\sigma|\nabla u(x)|^{2} .
$$

Here $W(u)$ is the coarse-grain free-energy and gives the energy when the density is uniform, while $\sigma>0$ is a constant related to the surface energy (cf. [4]).

If we assume that the fluid occupies a fixed container $\Omega$, with $\Omega$ an open region in $\mathbf{R}^{n}$, and that there are no other contributions to the energy, then the total energy $E(u)$ corresponding to a density distribution $u$ is given by

$$
E(u)=\int_{\Omega}\left\{W(u(x))+\sigma|\nabla u(x)|^{2}\right\} d x .
$$

Further, if the total mass of fluid in $\Omega$ is $m$, then the density distributions under consideration are subject to the constraint

$$
\int_{\Omega} u(x) d x=m .
$$

Our problem is to characterize the stable distributions of fluid in $\Omega$; that is, taking Gibb's definition of stability, we wish to characterize those density distributions $u$ that minimize $E(u)$ subject to the constraint (1.1).

What makes this problem interesting is that-for a two-phase fluid- $W(u)$ is not a convex function of $u$, but instead has the form shown in Fig. 1. Indeed, when $\sigma=0$ and the average density $r=m / \operatorname{vol}(\Omega)$ lies between $\alpha$ and $\beta$ (cf. Fig. 1), the global minimizers $u$ are piecewise-constant functions with $u(x) \equiv \alpha$ in some subset $\Gamma$ of $\Omega$ and $u(x) \equiv \beta$ in the remainder of $\Omega$. The volume of $\Gamma$ is determined by the constraint (1.1) anci must have a given value, $v_{0}$ say, but $\Gamma$ is otherwise arbitrary: any

\footnotetext{
*Received January 29, 1987.
} 
measurable set $\Gamma$ with volume $v_{0}$ generates-in the above sense-a global minimizer. Thus solutions can be very complicated, with an arbitrary number of transitions between $\alpha$ and $\beta$.

It is our purpose here to show-for regions of relatively simple shape-that the presence of "surface energy" through the additional term $\sigma|\nabla u|^{2}$ rules out such complicated behavior, even for arbitrarily small values of the constant $\sigma$.

For $n=1$ ( $\mathbf{R}^{n}$ the underlying space) a theorem of this type was obtained by Carr, Gurtin, and Slemrod [7], who showed that all local minimizers are monotone. This theorem was predated by work of Chafee [9], Casten and Holland [8], and Matano [16] for the unconstrained problem: Chafee proved that for $n=1$ all unconstrained local minimizers are constant; the other authors extended Chafee's result to arbitrary $n$, but convex or annular $\Omega$.
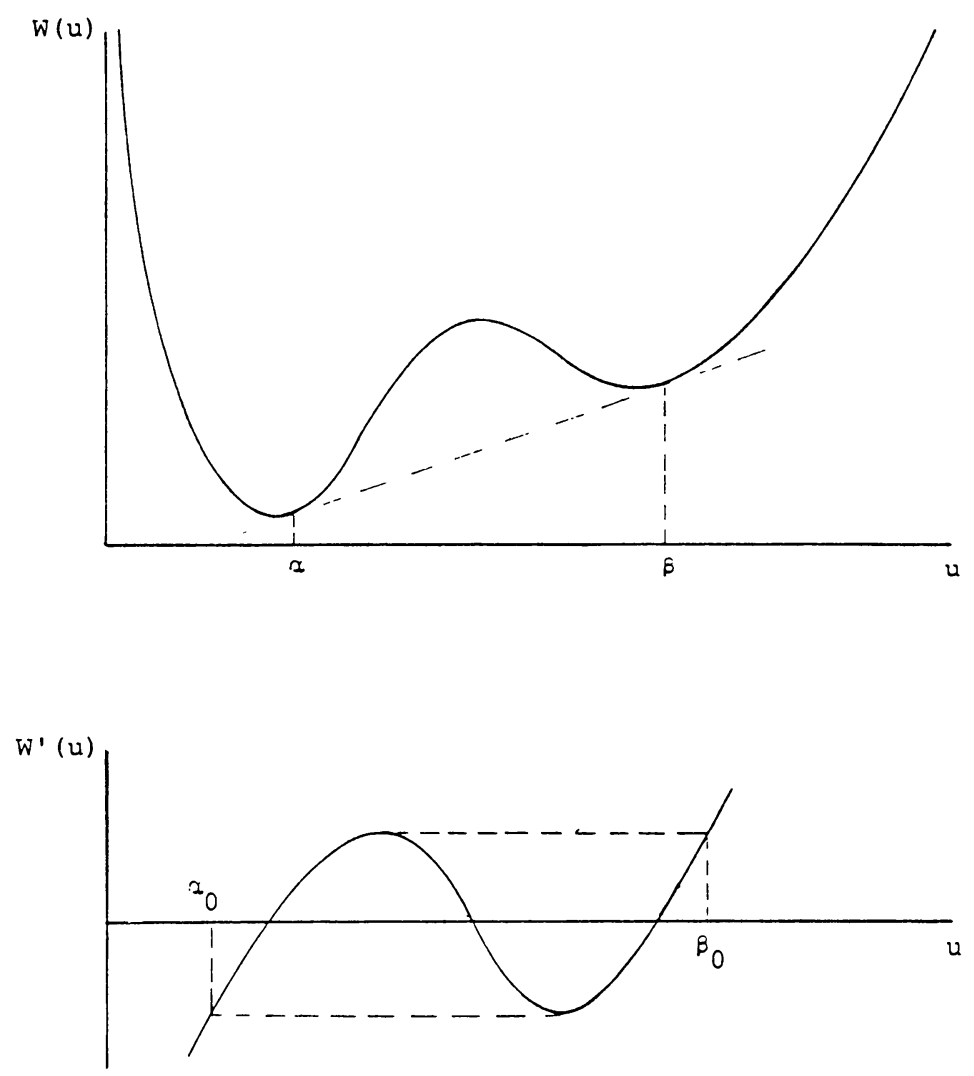

FIG. 1. The coarse-grain free-energy $W(u)$ and its derivative $W^{\prime}(u)$ for a two-phase fluid.

That results of this type cannot be expected for regions of arbitrary shape is clear from work of Matano [16], who established the existence of nonconstant local minimizers for the unconstrained problem in certain nonconvex regions. Matano's analysis extends to the constrained problem in regions $\Omega$ of the form shown in Fig. 2: for 
such regions this problem (with $\sigma$ small) can have local minimizers which are close to $\alpha$ in some compartments and close to $\beta$ in others, with the choice of compartments essentially arbitrary (cf. also [17, Example 6.6], [13]).

The first class of regions we consider are (not necessarily circular) cylinders. We show in Sec. 3 that for such regions all local minimizers are monotone in the axial direction.

In Sec. 4 we discuss regions with various degrees of rotational symmetry. We show that for balls, spherical shells, discs, and annuli, all rotationally-symmetric local minimizers are constant. We show further that:

(i) for $\Omega$ a disc or annulus and $u$ a local minimizer, the set on which $u_{\theta}>0$ is connected, as is the set with $u_{\theta}<0$;

(ii) for $\Omega$ a disc and $u$ a global minimizer, there is at least one direction in which $u$ is monotone.

We also show-for $\Omega$ an arbitrary bounded open set—that local minimizers belong to $L^{\infty}(\Omega)$.

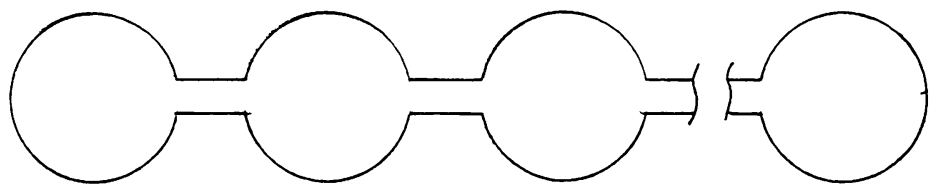

FIG. 2. A region $\Omega$ which can have minimizers close to $\alpha$ in some compartments and close to $\beta$ in others, with the choice of compartments essentially arbitrary.

In Sec. 5 we establish results which are valid not only for local minimizers but more generally for stationary points of $E$. Stationary points at which $E$ is not a local minimum correspond to unstable equilibria and might be important when studying corresponding evolution problems, that is, problems associated with the Cahn-Hilliard equation ([3], [5]). Indeed, preliminary numerical experiments seem to indicate that the system undergoes patterns qualitatively similar to unstable equilibria before settling down to a stable pattern. Moreover, within a given time span of experiments, it may happen that only unstable patterns are observed. Thus the study of the structure of nonminimal stationary points may, under certain circumstances, be of equal importance to that of local and global minimizers.

Stationary points $u$ of $E$ are solutions of the corresponding Euler-Lagrange equation and natural boundary condition:

$$
\Delta u=f(u) \quad \text { in } \Omega, \quad \partial u / \partial n=0 \quad \text { on } \partial \Omega .
$$

Here $f(u)=W^{\prime}(u)+\mu$, the constant $\mu$ being the Lagrange-multiplier corresponding to the constraint, while $\partial u / \partial n=\nabla u \cdot n$ with $n$ the outward unit normal on $\partial \Omega$. We prove that for $f^{\prime}$ bounded below and $\Omega=D \times G$ (or $\Omega=D$ ) with $D \subset \mathbf{R}^{p}$ sufficiently 
small, all solutions of $(\mathcal{N})$ are independent of the variables $\left(x_{1}, \ldots, x_{p}\right)$ that describe D.

Although we make no assumptions concerning the size of the constant $\sigma$, we shall never allow $\sigma=0$, nor will we study the limit $\sigma \rightarrow 0$; for that reason, we simplify the underlying equations by choosing length scales so that $\sigma=\frac{1}{2}$.

2. Existence and regularity. Some preliminary lemmas. We assume that the energy $W(u)$, the container $\Omega \subset \mathbf{R}^{n}$, and the total mass $m \in \mathbf{R}$ are prescribed. For convenience, we define the average density $r$ by $r=m /|\Omega|$, where $|\Omega|$ denotes the $n$-dimensional Lebesgue-measure of $\Omega$. To avoid repeated hypotheses we assume, once and for all, that

(A1) $\Omega$ is a bounded, open region with $C^{3}$ boundary, or the cartesian product of such regions;

(A2) $W \in C^{2+\gamma}\left(u_{0}, u_{1}\right)$ with $-\infty \leq u_{0}<u_{1} \leq+\infty$ and $0<\gamma<1$;

(A3) there exist numbers $a, b \in\left(u_{0}, u_{1}\right), a \leq r \leq b$, such that

$$
\sup _{\left(u_{0}, r\right)} W^{\prime} \leq \inf _{\left(b, u_{1}\right)} W^{\prime}, \quad \sup _{\left(u_{0}, a\right)} W^{\prime} \leq \inf _{\left(r, u_{1}\right)} W^{\prime} .
$$

Convention. We extend $W$ to all of $\mathbf{R}$ by defining $W(u)=+\infty$ for $u \notin\left(u_{0}, u_{1}\right)$.

Remarks. (i) We do not require that $m, u_{0}$, and $u_{1}$ be $\geq 0$, as there is no added difficulty in allowing negative values.

(ii) Condition (A3) is satisfied if, for example, $W$ is a convex function ( $W$ need not be strictly convex), or if $\left(u_{0}, u_{1}\right)=\mathbf{R}$ and

$$
\lim _{s \downarrow u_{0}} W^{\prime}(s)=-\infty, \quad \lim _{s \uparrow u_{1}} W^{\prime}(s)=+\infty .
$$

Condition (A3) will be used to derive an $L^{\infty}$ bound for local minimizers and to establish the existence of a global minimizer (see the two theorems of this section); this condition is not needed for any of our other results.

(iii) For the physical model illustrated in Fig. 1, (A3) is satisfied provided we take

$$
\begin{aligned}
& a=b=r \quad \text { when } r \leq \alpha_{0} \text { or } r \geq \beta_{0} ; \\
& a=\alpha_{0}, \quad b=\beta_{0} \quad \text { when } \alpha_{0}<r<\beta_{0} .
\end{aligned}
$$

(iv) Our hypotheses allow $W^{\prime}$ to be linear, so that the results in Secs. 3 and 4 apply also to the second eigenfunction of the Laplacian under Neumann boundary conditions.

By (A3),

$$
W(s) \geq-C_{1}-C_{2}|s| \quad(\forall s \in \mathbf{R}),
$$

with $C_{1}, C_{2} \geq 0$. It follows immediately from (2.2) and the boundedness of $\Omega$ that $E(u)$ is well defined on $H^{1}(\Omega)$, with values ranging in the interval $(-\infty,+\infty]$.

As noted in Sec. 1, we are concerned with the following variational problem: $(\mathscr{P})$ minimize

subject to

$$
E(u)=\int_{\Omega}\left\{\frac{1}{2}|\nabla u|^{2}+W(u)\right\}
$$

$$
\int_{\Omega} u=m
$$


We shall use the following terminology:

(i) $u$ is admissible if $u \in H^{1}(\Omega)$ and satisfies the constraint (2.4).

(ii) $u$ is a global minimizer if $u$ is admissible and $E(u) \leq E(v)$ for all admissible $v$.

(iii) $u$ is a local minimizer if $u$ is admissible and there is a neighborhood $\mathscr{U}$ of $u$ in $H^{1}(\Omega)$ such that $E(u) \leq E(v)$ for all admissible $v \in \mathscr{U}$.

THEOREM. There exists a global minimizer.

Proof. The Poincaré-Wirtinger inequality implies that

$$
\int_{\Omega}|\nabla u|^{2} \geq \lambda_{2}(\Omega)\left\{\int_{\Omega} u^{2}-m^{2} /|\Omega|\right\}
$$

for all admissible $u$, with $\lambda_{2}(\Omega)$ the lowest nonzero eigenvalue for $-\Delta$ (Neumann boundary conditions on $\partial \Omega$ ), and this inequality and (2.2) imply that, on the space of admissible functions, $E$ is bounded below with $E(u) \rightarrow+\infty$ as $\|u\|_{H^{1}(\Omega)} \rightarrow \infty$. Finally, (2.2) and Fatou's lemma imply that $E$ is weakly lower-semicontinuous on $H^{1}(\Omega)$. These observations and a standard argument yield the existence of a global minimizer.

The next result shows that local minimizers must take values between the constants $a$ and $b$ of (A3) (cf. 2.1).

THEOREM. Let $u$ be a local minimizer. Then

(i) $u \in L^{\infty}(\Omega)$; in fact,

$$
a \leq u(x) \leq b \text { for almost every } x \in \Omega ;
$$

(ii) $u \in H^{3}(\Omega) \cap C^{3+\gamma}(\Omega)$, and there is a constant $\mu$ such that

$$
\Delta u=W^{\prime}(u)+\mu \text { in } \Omega
$$

(iii) For any $\delta$ with $0<\delta<1, u$ is $C^{2+\delta}$ up to smooth subsurfaces of $\partial \Omega$ and satisfies

$$
\partial u / \partial n=0 \quad \text { on } \partial \Omega \text {. }
$$

Proof. Some simple terminology makes the proof transparent. Let $u \in H^{1}(\Omega)$. Then $c$ is a nontrivial value for $u$ if the sets

$$
\{x \in \Omega: u(x)>c\}, \quad\{x \in \Omega: u(x)<c\}
$$

have strictly-positive measure. Assume so, and let $v, w \in H^{1}(\Omega)$ be defined by

$$
v(x)=\max \{u(x), c\}, \quad w(x)=\min \{u(x), c\} ;
$$

then $v$ is $u$ chopped below at $c$, while $w$ is $u$ chopped above at $c$.

Consider (i) and let $a \leq r$ be as in (A3). Let $u$ be a local minimizer and assume that $u<a$ on a set of nonzero measure. Then, by the constraint, $a$ and $r$ are nontrivial values of $u$. A further consequence of the constraint is that $u$ remains a local minimizer when a linear function is added to $W$, or equivalently, when a constant is added to $W^{\prime}$; thus, in view of the second of (A3), we may, without loss in generality, assume that

$$
W^{\prime} \leq 0 \quad \text { on }\left(u_{0}, a\right), \quad W^{\prime} \geq 0 \quad \text { on }\left(r, u_{1}\right) .
$$


Choose $\varepsilon>0$. Then there are nontrivial values $a_{\varepsilon}$ and $r_{\varepsilon}$ of $u$ with $a_{\varepsilon} \in\left(u_{0}, a\right)$, $r_{\varepsilon} \in\left(r, u_{1}\right)$ such that, letting $u_{\varepsilon}$ denote the function $u$ chopped below at $a_{\varepsilon}$ and above at $r_{\varepsilon}$

$$
\int_{\Omega} u_{\varepsilon}=\int_{\Omega} u, \quad\left\|u-u_{\varepsilon}\right\|_{H^{1}(\Omega)}<\varepsilon .
$$

Moreover,

$$
\int_{\Omega}\left|\nabla u_{\varepsilon}\right|^{2}<\int_{\Omega}|\nabla u|^{2}, \quad \int_{\Omega} W\left(u_{\varepsilon}\right) \leq \int_{\Omega} W(u),
$$

with the latter relation a direct consequence of (2.10). This yields $E\left(u_{\varepsilon}\right)<E(u)$, a contradiction since $\varepsilon$ was arbitrarily chosen. A similar contradiction arises when $u>b$ on a set of nonzero measure.

The results (ii) and (iii) are direct consequences of the following: (a) the $L^{\infty}$ estimate of (i); (b) the weak Euler-Lagrange equation for (2.3) and (2.4), namely,

$$
\int_{\Omega}\left\{\nabla u \cdot \nabla \eta+W^{\prime}(u) \eta+\mu \eta\right\}=0 \quad\left(\forall \eta \in H^{1}(\Omega)\right)
$$

with $\mu$ a Lagrange multiplier; (c) the Regularity Lemma which we state below and prove in the Appendix; (d) standard regularity theory.

Regularity Lemma. ${ }^{1}$ Let $g \in H^{1}(\Omega)$ and suppose that $u \in H^{1}(\Omega)$ satisfies

$$
\int_{\Omega} \nabla u \cdot \nabla \eta=\int_{\Omega} g \eta \quad\left(\forall \eta \in H^{1}(\Omega)\right) .
$$

Then $u \in H^{3}(\Omega)$.

Remarks. (i) It is clear that (i) of the above theorem requires only that $\Omega$ be a bounded open set.

(ii) Consider the model illustrated in Fig. 1. In view of (2.5) and Remark (iii) given at the beginning of the section, for $r \leq \alpha_{0}$ or $r \geq \beta_{0}$ the only local minimizer is the constant $u(x) \equiv r$.

(iii) The usual method of proving the above theorem is based on an assumption of the form

$$
\left|W^{\prime}(u)\right| \leq C_{1}+C_{2}|u|^{p}
$$

with $C_{1}, C_{2} \geq 0$ and $1 \leq p<(n+2) /(n-2)$. Note that the standard example $W(u)=c u^{4}+d u^{2}$ violates this assumption when $n \geq 4$, while our assumption (A3) covers this case.

We use the term variation for any function $\varphi \in H^{1}(\Omega)$ that satisfies

$$
\int_{\Omega} \varphi=0
$$

and, for $u \in H^{1}(\Omega)$ and $\varphi$ a variation, we write

$$
J(u)(\varphi)=\int_{\Omega}\left\{|\nabla \varphi|^{2}+W^{\prime \prime}(u) \varphi^{2}\right\} .
$$

\footnotetext{
'Assumption (A1) is tacit. While the Regularity Lemma is standard for smooth domains, it does not seem to be known for cartesian products of smooth domains, which are of interest here.
} 
The function $J(u)(\cdot)$ is the second variation of $E$ at $u$ and, as is well known, for $u$ a local minimizer and $\varphi$ a variation,

$$
J(u)(\varphi) \geq 0 .
$$

We close this section with some useful definitions and identities. For any function $\varphi \in H^{1}(\Omega)$ we write

$$
\Omega^{+}(\varphi)=\{x \in \Omega: \varphi(x)>0\}, \quad \Omega^{-}(\varphi)=\{x \in \Omega: \varphi(x)<0\},
$$

and define

$$
\varphi^{ \pm}=\frac{1}{2}\{\varphi \pm|\varphi|\}
$$

Then

$$
\varphi=\varphi^{+}+\varphi^{-}, \quad \varphi^{+} \geq 0, \quad \varphi^{-} \leq 0, \quad \varphi^{ \pm} \in H^{1}(\Omega) .
$$

We will repeatedly use the following version of Green's formula (cf., e.g., [11; Lemma 1.5.3.7]): for $w \in H^{2}(\Omega), v \in H^{1}(\Omega)$ (and $\Omega$ consistent with (A1)),

$$
\int_{\Omega}(\Delta w) v=-\int_{\Omega}(\nabla w \cdot \nabla v)+\int_{\partial \Omega} v(\partial w / \partial n) .
$$

This formula is valid, since the cartesian product of smooth domains has a Lipschitz boundary.

Finally, we note that if $\Omega=D \times G, D \subset \mathbf{R}^{p}$, and if $g$ is $C^{2}$ up to smooth subsurfaces of $\partial \Omega$, then $\partial g / \partial n=0$ on $\partial \Omega$ implies

$$
\frac{\partial}{\partial n}\left(\frac{\partial g}{\partial x_{k}}\right)=0 \text { on } D \times \partial G \text { for } k=1,2, \ldots, p \text {. }
$$

Indeed, (2.21) is a direct consequence of the fact that, on $D \times \partial G$ for $k=1,2, \ldots, p$, $\partial / \partial x_{k}$ is a tangential derivative, while the normal $n$ is independent of $\left(x_{1}, x_{2}, \ldots, x_{p}\right)$.

3. Structure of local minimizers for cylinders. In this section we shall discuss the behavior of local minimizers when the domain $\Omega$ is a cylinder in $\mathbf{R}^{n}$. For convenience, we let the $x_{1}$-coordinate define the axis of the cylinder; then $\Omega=(0, L) \times G$ with $L>0$ and $G \subset \mathbf{R}^{n-1}$.

TheOREM. Let $\Omega$ be a cylinder. Then all local minimizers are monotone in the axial direction.

Proof. Let $u$ be a local minimizer and define $\varphi=\partial u / \partial x_{1}$, so that, by (2.6),

$$
\Delta \varphi=W^{\prime \prime}(u) \varphi .
$$

Further, let $\partial \Omega=\mathscr{E} \cup \mathscr{S}$ with $\mathscr{E}=\{0, L\} \times G$ the end faces and $\mathscr{S}=(0, L) \times \partial G$ the lateral surface. Then, because of (2.7) and (2.21), $\varphi$ satisfies the boundary conditions

$$
\varphi=0 \quad \text { on } \mathscr{E}, \quad \partial \varphi / \partial n=0 \quad \text { on } \mathscr{S} .
$$

Assume that $u(x)$ is not monotone in $x_{1}$. Then $\varphi$ changes sign on $\Omega$, and neither of $\varphi^{ \pm}$(cf. (2.18)) is identically zero; hence we may define $\varphi^{*} \in H^{1}(\Omega)$ through

$$
\varphi^{*}=\varphi^{+}+\alpha \varphi^{-}
$$


with $\alpha$ chosen so that

$$
\int_{\Omega} \varphi^{*}=0
$$

Let

$$
\varphi^{* *}=\varphi^{+}+\alpha^{2} \varphi^{-}
$$

then, by (3.2),

$$
\varphi^{*}=\varphi^{* *}=0 \quad \text { on } \mathscr{E} \text {. }
$$

Also,

$$
\left(\varphi^{*}\right)^{2}=\varphi \varphi^{* *}, \quad\left|\nabla \varphi^{*}\right|^{2}=\nabla \varphi \cdot \nabla \varphi^{* *}
$$

we may therefore use (2.15), (3.1), and Green's formula (2.20) to conclude that

$$
J(u)\left(\varphi^{*}\right)=\int_{\partial \Omega} \varphi^{* *} \frac{\partial \varphi}{\partial n}
$$

and hence, by (3.2) and (3.6), that

$$
J(u)\left(\varphi^{*}\right)=0 .
$$

Thus and by $(2.16), \varphi^{*}$ minimizes $J(u)(\psi)$ over all variations $\psi$; hence $\varphi^{*}$ satisfies

$$
\begin{gathered}
\Delta \varphi^{*}=W^{\prime \prime}(u) \varphi^{*}+\text { constant } \text { in } \Omega, \\
\partial \varphi^{*} / \partial n=0 \text { on } \partial \Omega .
\end{gathered}
$$

By (3.1) and (3.9),

$$
\Delta \varphi^{*}=W^{\prime \prime}(u) \varphi^{*} \quad \text { in } \Omega .
$$

Thus, in view of (3.6) and (3.10), $\varphi^{*}$ satisfies a second-order linear elliptic equation in $\Omega$ and is consistent with Dirichlet and Neumann boundary conditions-both nullon $\mathscr{E}$. We may therefore use the unique continuation theorem (cf. [6]) to conclude that $\varphi^{*}$ and (hence) $\varphi$ vanish identically. This contradiction completes the proof.

4. Structure of minimizers in regions with rotational symmetry. For $\Omega$ a ball, each spherically symmetric field on $\Omega$ is necessarily constant on $\partial \Omega$. Similarly, on a cylinder a field with cylindrical symmetry is constant on the boundary of each cross-section perpendicular to the axis of the cylinder. The next result is a general proposition for fields of this type. For convenience, let us agree to call a function $\psi$ locally constant on a set $A$ if $\psi$ is constant on each connected component of $A$.

THEOREM. Let $u$ be a local minimizer.

(i) If $u$ is locally constant on $\partial \Omega$, then $u$ is constant in $\Omega$.

(ii) If $\Omega=B \times G, B \subset \mathbf{R}^{p}, 2 \leq p \leq n-1$, and if, for each $\left(x_{p+1}, \ldots, x_{n}\right) \in G, u(x)$, as a function of $\left(x_{1}, \ldots, x_{p}\right)$, is locally constant on $\partial B$, then $u(x)$ is independent of $\left(x_{1}, \ldots, x_{p}\right)$.

Proof. We shall prove only (ii). The proof of (i) is completely analogous.

For $x$ in the closure of $\Omega$, we write $x=\left(x_{B}, x_{G}\right), x_{B} \in B, x_{G} \in G$. Choose $k$, $1 \leq k \leq p$, let $\varphi=\partial u / \partial x_{k}$, and let $n_{k}$ denote the $k$ th component of the outward unit normal $n$ on $\partial \Omega$. Then, since $n_{k}=0$ on $B \times \partial G$, while $u\left(x_{B}, x_{G}\right)$, for each fixed $x_{G}$, is locally constant in $x_{B}$ on $\partial B$,

$$
\int_{\Omega} \varphi=\int_{\partial \Omega} u n_{k}=\int_{G} \int_{\partial B} u n_{k}=0,
$$


and $\varphi$ is a variation.

Next, on $\partial B \times G, \varphi=\partial u / \partial x_{k}$ is a linear combination of tangential and normal derivatives of $u\left(x_{B}, x_{G}\right)$ with respect to $x_{B}$ on $\partial B$. But the former vanishes because $u$ is locally constant on $\partial B$, and the latter vanishes because of the natural boundary condition $(2.7)$; hence

$$
\varphi=0 \text { on } \partial B \times G \text {. }
$$

Further, by (2.21), $\partial \varphi / \partial n=0$ on $B \times \partial G$. Thus (2.15), (3.1), (2.20), and (4.1) imply that $J(u)(\varphi)=0$; hence arguing as in Sec. 3, we conclude that $\varphi \equiv 0$.

This theorem has an obvious counterpart for balls $\left\{x: 0 \leq|x|<r_{1}\right\}$ and for spherical shells $\left\{x: 0<r_{0}<|x|<r_{1}\right\}$. (In $\mathbf{R}^{2}$, ball=disc, spherical shell=annulus.)

Corollary. Let $u$ be a local minimizer.

(i) If $\Omega$ is a ball or a spherical shell, and if $u(x)$ depends on $x$ only through $|x|$, then $u$ is constant.

(ii) If $\Omega=B \times G, B \subset \mathbf{R}^{p}, 2 \leq p \leq n-1$, with $B$ a ball or a spherical shell, and if $u(x)$ depends on $\left(x_{1}, \ldots, x_{p}\right)$ only through its absolute value, then $u(x)$ is independent of $\left(x_{1}, \ldots, x_{p}\right)$.

We now limit our attention to $\mathbf{R}^{2}$ and, in particular, assume that

$$
\bar{\Omega}=\left\{(r, \theta): r_{0} \leq r \leq r_{1}, 0 \leq \theta<2 \pi\right\}
$$

with $(r, \theta)$ polar coordinates, so that $\Omega$ is a disc $\left(r_{0}=0\right)$ or an annulus $\left(r_{0}>0\right)$. Let $u_{\theta}=\partial u / \partial \theta, u_{r}=\partial u / \partial r$. From the corollary, if $u$ with $u_{\theta} \equiv 0$ is a local minimizer, then $u$ is constant. The next theorem deals with the more interesting case $u_{\theta} \not \equiv 0$; there we use the notation (2.17).

TheOREM. Let $\Omega \subset \mathbf{R}^{2}$ be a disc or annulus, and let $u$ be a local minimizer with $u_{\theta} \not \equiv 0$. Then

(i) each of the sets $\Omega^{ \pm}\left(u_{\theta}\right)$ is connected;

(ii) on each of the circles comprising $\partial \Omega, u$ has exactly one local maximum and exactly one local minimum.

Proof. (i) Since $u(r, 0)=u(r, 2 \pi)$, it is clear that each of the sets $\Omega^{ \pm}=\Omega^{ \pm}\left(u_{\theta}\right)$ is nonempty. Choose a connected component $\Gamma^{+}$from $\Omega^{+}$and a connected component $\Gamma^{-}$from $\Omega^{-}$, and define

$$
\Lambda=\Omega \backslash\left(\Gamma^{+} \cup \Gamma^{-}\right) \text {. }
$$

To prove that $\Omega^{ \pm}$are each connected, it suffices to show that $\dot{\Lambda}=\varnothing$.

With this in mind, let

$$
\varphi^{*}(r, \theta)= \begin{cases}u_{\theta}(r, \theta), & (r, \theta) \in \Gamma^{+}, \\ \alpha u_{\theta}(r, \theta), & (r, \theta) \in \Gamma^{-}, \\ 0, & (r, \theta) \in \Lambda,\end{cases}
$$

with $\alpha>0$ chosen so that $\int_{\Omega} \varphi^{*}=0$. Then steps exactly analogous to those used in Sec. 3 lead to the conclusion that $\varphi^{*}$ satisfies (3.11). Thus $\dot{\Lambda} \neq \varnothing$ implies $\varphi^{*} \equiv 0$ in $\Omega$, a contradiction; hence $\dot{\Lambda}=\varnothing$.

(ii) Since $\varphi^{*}$-as defined above-satisfies (3.10) and (3.11), the zeros of $\varphi^{*}$ on $\partial \Omega$ form a nowhere dense subset of $\partial \Omega$; hence (i) implies (ii). 
Remark. By the theorem of Hartman and Wintner [12], the zeros of $\varphi^{*}\left(=u_{\theta}\right)$ form a locally finite family of $C^{1}$ curves. Combining this result with the above theorem, we can further show that the set of zeros of $\varphi^{*}$ is a simple $C^{1}$ curve for $\Omega$ a disc and is a disjoint pair of simple $C^{1}$ curves for $\Omega$ an annulus. Moreover, using Kelvin's transformation and the condition $\partial \varphi^{*} / \partial n=0$ on $\partial \Omega$, we see that these curves intersect $\partial \Omega$ orthogonally. In particular, $\varphi^{*}$ has exactly two zeros on each of the circles comprising $\partial \Omega$.

THEOREM. Let $u$ be a global minimizer with $\Omega \subset \mathbf{R}^{2}$ a disc. Then $\Omega$ has a diameter $P Q$ such that

(i) $u$ is symmetric with respect to $P Q$;

(ii) $u$ is monotone in the direction parallel to $P Q$;

(iii) $u_{\theta} \geq 0$ on one side of $P Q, u_{\theta} \leq 0$ on the other side;

(iv) $u$ takes its maximum at one of $P, Q$ and its minimum at the other.

Proof. The existence of a diameter $P Q$ consistent with (i) and (iii) follows from the theory of symmetric rearrangement (cf. [14], [15]).

More precisely, let $u^{*}$ be a circular rearrangement of $u$; that is, $u^{*}$ is a measurable function on $\Omega$ with the following properties:

(i) $u^{*}$ is symmetric with respect to some diameter $P^{\prime} Q^{\prime}$ of $\Omega$;

(ii) $u_{\theta}^{*} \geq 0$ on one side of $P^{\prime} Q^{\prime} ; u_{\theta}^{*} \leq 0$ on the other;

(iii) for each $\alpha \in \mathbf{R}$ and $r \in\left(0, r_{1}\right]$,

$$
\mu_{r}\left(\left\{x \in C_{r}: u^{*}(x)>\alpha\right\}\right)=\mu_{r}\left(\left\{x \in C_{r}: u(x)>\alpha\right\}\right),
$$

where $C_{r}=\left\{x \in \mathbf{R}^{2}:|x|=r\right\}$ and $\mu_{r}$ is one-dimensional Lebesgue measure on $C_{r}$. Condition (iii) asserts that $u^{*}$ and $u$ are equimeasurable on each circle $C_{r}$ and hence also on $\Omega$. Thus by standard results in the theory of rearrangements (cf. [14] and references therein)

$$
\int_{\Omega}\left|\nabla u^{*}\right|^{2} \leq \int_{\Omega}|\nabla u|^{2} \quad \text { and } \quad \int_{\Omega} W\left(u^{*}\right)=\int_{\Omega} W(u) ;
$$

hence

$$
E\left(u^{*}\right) \leq E(u) .
$$

But $u$ is a global minimizer; thus $E\left(u^{*}\right)=E(u)$ and

$$
\int_{\Omega}\left|\nabla u^{*}\right|^{2}=\int_{\Omega}|\nabla u|^{2}
$$

Thus, by [14, Corollary 2.35], $u=u^{*}$ modulo rotation. In fact, to verify that Corollary 2.35 of [14] applies, we must check the following: (a) $u \in C^{1}(\bar{\Omega})$; (b) there is a set $N \subset\left[0, r_{1}\right]$ of measure zero such that for each $r \in\left[0, r_{1}\right] \backslash N$, the set $\left\{\theta: u_{\theta}(r, \theta)=0\right\}$ is finite. (Although Corollary 2.35 requires that $u$ be analytic, a careful study of the proof shows that the above conditions are sufficient.) Condition (a) is satisfied since $u$ satisfies the Euler-Lagrange equation (2.6). To prove (b) it suffices to show that there is a set $N \subset\left[0, r_{1}\right]$ of measure zero such that, for each $r \in\left[0, r_{1}\right] \backslash N$, the circle $C_{r}$ and the $C^{1}$ curve $\left\{x \in \Omega: u_{\theta}(x)=0\right\}$ intersect transversally. But this is an easy consequence of Sard's theorem. Hence we may apply [14, Corollary 2.35] to verify that $u=u^{*}$ modulo rotation, which proves (i) and (iii). 
Choose coordinates so that $P Q$ is parallel to the $x_{2}$-axis and let $\varphi=\partial u / \partial x_{2}$. Then, by (iii) and (2.7), $\varphi \geq 0$ on $\partial \Omega$, so that, using the notation (2.17),

$$
\varphi=0 \text { on } \partial \Omega \cap \partial \Omega^{-}(\varphi) .
$$

To establish (ii) it suffices to show that $\varphi$ does not change sign in $\Omega$. Assume, to the contrary, that $\varphi$ changes sign in $\Omega$, and let $\varphi^{*}$ be as defined in (3.3). Then, since $u$ is a local minimizer, we conclude, with the aid of (3.5), (3.8), and (4.2), that

$$
0 \leq J(u)\left(\varphi^{*}\right)=\int_{\partial \Omega} \varphi \frac{\partial \varphi}{\partial n} .
$$

Let $\psi=\partial u / \partial x_{1}$, so that, by (i), $\int_{\Omega} \psi=0$ and $\psi$ is a variation; hence, using (2.15) and (3.1) (with $\varphi$ replaced by $\psi$ ),

$$
0 \leq J(u)(\psi)=\int_{\partial \Omega} \psi \frac{\partial \psi}{\partial n} .
$$

Thus, in view of $(4.3)$ and the identity $\varphi^{2}+\psi^{2}=|\nabla u|^{2}$, it follows that

$$
0 \leq J(u)\left(\varphi^{*}\right)+J(u)(\psi)=\frac{1}{2} \int_{\partial \Omega} \frac{\partial}{\partial n}|\nabla u|^{2} .
$$

On the other hand, since $\Omega$ is a disc of radius $r_{1}$ and $\partial u / \partial n=0$ on $\partial \Omega$, we have the identity

$$
\frac{\partial}{\partial n}|\nabla u|^{2}=-\frac{2}{r_{1}}|\nabla u|^{2} \quad \text { on } \partial \Omega \text {. }
$$

Clearly, (4.3)-(4.6) are compatible only if

$$
\begin{gathered}
\nabla u=0 \quad \text { on } \partial \Omega, \\
J(u)\left(\varphi^{*}\right)=0 .
\end{gathered}
$$

By (4.7), $\varphi^{*}=0$ on $\partial \Omega$; thus (4.8) and an argument analogous to that used in Sec. 3 imply that $\varphi^{*}$ and (hence) $\varphi$ vanish in $\Omega$. This contradiction proves (ii).

Assertion (iv) follows at once from (ii) and (iii).

5. Structure of stationary fields for bodies with small dimensions. In this section we discuss stationary fields, that is, solutions $u$ of the elliptic Neumann problem:

$$
\Delta u=f(u) \quad \text { in } \Omega, \quad \frac{\partial u}{\partial n}=0 \quad \text { on } \partial \Omega .
$$

Problem $(\mathscr{N})$ characterizes critical points of the function $E$ over $H^{1}(\Omega)$, in which case $f=W^{\prime}$, and critical points of $E$ over

$$
\left\{u \in H^{1}(\Omega): \int_{\Omega} u=m\right\},
$$

in which case $f=W^{\prime}+\mu$ with $\mu$ a Lagrange multiplier. For the results discussed here the particular form of $W$ is irrelevant; aside from a certain boundedness assumption, we need only assume that

$$
f \in C^{1}\left(u_{0}, u_{1}\right) \text {. }
$$

(The term solution of $(\mathcal{N})$ will be reserved for solutions $u \in H^{3}(\Omega)$ with $u_{0}<u(x)<$ $u_{1}$ for all $x \in \Omega$.) 
We begin with a result which shows that-for inf $f^{\prime}$ not too negative-all solutions of $(\mathscr{N})$ are constant. To state this and future results precisely, let $0=\lambda_{1}(D)<$ $\lambda_{2}(D) \leq \lambda_{3}(D) \leq \cdots$ denote the eigenvalues of the problem

$$
\Delta v+\lambda v=0 \quad \text { in } D, \quad \partial v / \partial n=0 \quad \text { on } \partial D .
$$

The following proposition seems well known, but we could not find a reference; for completeness we present a proof.

Proposition. ${ }^{2}$ Let inf $f^{\prime}>-\lambda_{2}(\Omega)$. Then all solutions of $(\mathscr{N})$ are constant.

Proof. Let $u$ satisfy $(\mathcal{N})$. Then

$$
\int_{\Omega} f(u)=0 .
$$

If $f(u(x))=0$ for: all $x \in \Omega$, then, by $(\mathcal{N}), u$ is constant. We may therefore assume that $f(u(x)) \not \equiv 0$. Then there is a $c \in\left(u_{0}, u_{1}\right)$ and points $x_{1}, x_{2} \in \Omega$ such that

$$
f(c)=0, \quad u\left(x_{1}\right)<c<u\left(x_{2}\right) .
$$

Put $\varphi=u-c$ and define $\varphi^{*}$ and $\varphi^{* *}$ through (3.3)-(3.5). (Since neither of the functions $\varphi^{ \pm}$vanishes identically, (3.4) defines $\alpha \in \mathbf{R}$.) Then, by (3.4),

$$
\int_{\Omega}\left|\nabla \varphi^{*}\right|^{2} \geq \lambda_{2}(\Omega) \int_{\Omega} \varphi^{* 2}
$$

Thus, since $\partial \varphi^{* *} / \partial n=0$ on $\partial \Omega,(3.7)$ yields

$$
\begin{aligned}
-\int_{\Omega} \varphi^{* *} f(u) & =-\int_{\Omega} \varphi^{* *} \Delta u=\int_{\Omega}\left|\nabla \varphi^{*}\right|^{2} \geq \lambda_{2}(\Omega) \int_{\Omega}\left(\varphi^{*}\right)^{2}, \\
& -\varphi^{* *} f(u) \leq k \varphi^{* *} \varphi=k\left(\varphi^{*}\right)^{2},
\end{aligned}
$$

where $k=-\inf f^{\prime} ;$ hence

$$
k \int_{\Omega}\left(\varphi^{*}\right)^{2} \geq \lambda_{2}(\Omega) \int_{\Omega}\left(\varphi^{*}\right)^{2} .
$$

But $k<\lambda_{2}(\Omega)$. Thus $\int_{\Omega}\left(\varphi^{*}\right)^{2}=0$, so that $u \equiv c$.

The foregoing proposition would be easier to apply if the hypothesis on inf $f^{\prime}$ were expressed in terms of $\operatorname{diam}(\Omega)$, the maximum diameter of $\Omega$, rather than in terms of $\lambda_{2}(\Omega)$. For that reason we state the following estimate of Payne and Weinberger [18] (cf. [2, Theorem 3.24]): for any convex domain $D \subset \mathbf{R}^{n}$,

$$
\lambda_{2}(D) \geq \pi^{2} / \operatorname{diam}(D)^{2} .
$$

Using (5.2) we can show that-for $f^{\prime}$ bounded below ${ }^{3}$ and $\Omega$ convex and small-all solutions of $(\mathscr{N})$ are constant.

Corollary. Let $\Omega$ be convex and assume that

$$
\pi^{2} / \operatorname{diam}(\Omega)^{2}>-\inf f^{\prime} .
$$

${ }^{2} \mathrm{Cf}$. [10], who establish a general result for systems, a result which, when specialized to a single equation, asserts that if $\sup \left|f^{\prime}\right|<\lambda_{2}(\Omega)$, then all solutions of $(\mathscr{N})$ are constant.

${ }^{3}$ Note that for the physical model described in Fig. $1, f^{\prime}=W^{\prime \prime}$ is bounded below. 
Then all solutions of $(\mathscr{N})$ are constant.

We now extend this result to regions $\Omega$ of the form

$$
\Omega=D \quad \text { or } \quad \Omega=D \times G, \quad \partial D \text { and } \partial G \text { of class } C^{3} ;
$$

we show that for $D$ small, solutions $u$ of $(\mathcal{N})$ are independent of position in $D$. THEOREM. Suppose that $\Omega$ has the form (5.3) and that

$$
\lambda_{2}(D)>-\inf f^{\prime}
$$

with $p$ the dimension of $D$. Then each solution $u\left(x_{1}, x_{2}, \ldots, x_{n}\right)$ of $(\mathcal{N})$ is independent of $\left(x_{1}, x_{2}, \ldots, x_{p}\right)$.

Proof. Let $\nabla_{p}$ and $\Delta_{p}$, respectively, denote the gradient and Laplacian with respect to $\left(x_{1}, \ldots, x_{p}\right)$ holding $\left(x_{p+1}, \ldots, x_{n}\right)$ fixed. Further, let $\left\{v_{k}\right\}$ be an orthonormal system of eigenfunctions for (5.1), with $v_{k}$ corresponding to the eigenvalue $\lambda_{k}=\lambda_{k}(D)$. Fix $\left(x_{p+1}, \ldots, x_{n}\right)$ in $G$ and consider $u(x)$ as a function $\hat{u}\left(x_{1}, \ldots, x_{p}\right)$ of $\left(x_{1}, \ldots, x_{p}\right) \in D$. Then, since $\partial u / \partial n=0$ on $\partial \Omega$, it follows that

$$
\partial \hat{u} / \partial n=0 \text { on } \partial D .
$$

Writing $\hat{u}=\sum_{k=1}^{\infty} c_{k} v_{k}$ for the eigenfunction expansion of $\hat{u}$, and using (5.5) and the fact that $\lambda_{1}=0$, we find that

$$
\int_{D}\left(\Delta_{p} \hat{u}\right)^{2}=\sum_{k=2}^{\infty} c_{k}^{2} \lambda_{k}^{2}, \quad \int_{D}\left|\nabla_{p} \hat{u}\right|^{2}=-\int_{D} \hat{u} \Delta_{p} \hat{u}=\sum_{k=2}^{\infty} c_{k}^{2} \lambda_{k},
$$

and hence that

$$
\int_{D}\left(\Delta_{p} \hat{u}\right)^{2} \geq \lambda_{2}(D) \int_{D}\left|\nabla_{p} \hat{u}\right|^{2} .
$$

Let $\hat{\psi}_{i}=\partial \hat{u} / \partial x_{i}, i=1,2, \ldots, p$. Then, by $(5.5)$,

$$
\begin{aligned}
\int_{D}\left(\Delta_{p} \hat{u}\right)^{2} & =-\int_{D} \nabla_{p} \hat{u} \cdot \nabla_{p}\left(\Delta_{p} \hat{u}\right)=-\sum_{i=1}^{p} \int_{D} \hat{\psi}_{i} \Delta_{p} \hat{\psi}_{i} \\
& =\sum_{i=1}^{p}\left\{-\int_{\partial D} \hat{\psi}_{i} \frac{\partial \hat{\psi}_{i}}{\partial n}+\int_{D}\left|\nabla_{p} \hat{\psi}_{i}\right|^{2}\right\} .
\end{aligned}
$$

Next, let $\psi_{i}=\partial u / \partial x_{i}, i=1,2, \ldots, p$. Then, by $(2.21), \partial \psi_{i} / \partial n=0$ on $D \times \partial G$, and we may conclude from this and $(\mathscr{N})$ that

$$
\begin{aligned}
\sum_{i=1}^{p} \int_{\Omega}\left|\nabla_{p} \psi_{i}\right|^{2} & \leq \sum_{i=1}^{p} \int_{\Omega}\left|\nabla \psi_{i}\right|^{2}=\sum_{i=1}^{p}\left\{\int_{\partial \Omega} \psi_{i} \frac{\partial \psi_{i}}{\partial n}-\int_{\Omega} \psi_{i} \Delta \psi_{i}\right\} \\
& =\sum_{i=1}^{p} \int_{\partial D \times G} \psi_{i} \frac{\partial \psi_{i}}{\partial n}-\int_{\Omega} f^{\prime}(u)\left|\nabla_{p} u\right|^{2} .
\end{aligned}
$$

Combining (5.7) and (5.8), we get

$$
\int_{\Omega}\left(\Delta_{p} u\right)^{2} \leq-\int_{\Omega} f^{\prime}(u)\left|\nabla_{p} u\right|^{2} \leq\left(-\inf f^{\prime}\right) \int_{\Omega}\left|\nabla_{p} u\right|^{2},
$$

and this, (5.4), and (5.6) yield the desired conclusion. 
Remarks. (i) In view of (5.2), when $D$ is convex the condition (5.4) may be replaced by

$$
\pi^{2} / \operatorname{diam}(D)^{2}>-\inf f^{\prime} .
$$

(ii) A typical example to which the above theorem applies is a cylindrical region of the form $\Omega=(0, L) \times G$. The conclusion of the theorem may then be interpreted as follows: (a) for a thin plate (i.e., for $L$ sufficiently small) stationary states are independent of the length variable $x_{1} \in(0, L)$; (b) for a thin $\operatorname{rod}$ (i.e., for $G$ sufficiently small) stationary states depend only on $x_{1}$.

Acknowledgment. We gratefully acknowledge H. F. Weinberger for useful discussions. This work was supported in part (MEG) by the Army Research Office and the National Science Foundation.

Appendix. Proof of the Regularity Lemma. The conclusion of the Regularity Lemma is well known for regions $\Omega$ with $\partial \Omega$ of class $C^{3}$; we therefore confine our attention to product domains $\Omega=D \times G$.

Let $D$ be a bounded domain in $\mathbf{R}^{p}$ with $\partial D$ of class $C^{3}$. Further, let $0=\lambda_{1}<$ $\lambda_{2} \leq \lambda_{3} \leq \cdots$ denote-for the Neumann problem-the eigenvalues of $-\Delta$ on $D$, and let $\left\{\varphi_{i}\right\}$ designate a corresponding $L^{2}(D)$-orthonormal system of eigenfunctions. Such a system exists and forms an orthonormal basis for $L^{2}(D)$; for convenience, we call $\left\{\lambda_{i}, \varphi_{i}\right\}$ a Neumann system for $D$. Each function $g \in L^{2}(D)$ admits the unique expansion

$$
g=\sum c_{i} \varphi_{i}
$$

where the $c$ 's are real numbers; for convenience, we write $s_{k}(g)$ for the formal sum

$$
s_{k}(g)=\sum\left(1+\lambda_{i}\right)^{k} c_{i}^{2} .
$$

The following additional notation will be useful:

$$
N^{1}(D)=H^{1}(D) ; \quad N^{k}(D)=\left\{g \in H^{k}(D): \partial g / \partial n=0 \text { on } \partial D\right\}, \quad k=2,3 ;
$$

given positive functions $r, s$ on a set $A$, we say that $r$ is equivalent to $s$ on $A$ if there exist constants $C_{1}, C_{2}>0$ such that

$$
r(a) \leq C_{1} s(a) \leq C_{2} r(a) \quad(\forall a \in A) .
$$

Lemma 1. Choose $k \in\{1,2,3\}$. Then

(i) given $g=\sum c_{i} \varphi_{i} \in L^{2}(D)$,

$$
g \in N^{k}(D) \Leftrightarrow s_{k}(g)<\infty ;
$$

(ii) $\|\cdot\|_{H^{k}(D)}$ is equivalent to $\left(s_{k}\right)^{1 / 2}$ on $N^{k}(D)$.

Proof. Since $\Delta \varphi_{i}=\lambda_{i} \varphi_{i}$, we may use Green's formula to derive the following relation between the inner products on $L^{2}(D)$ and $H^{1}(D)$ :

$$
\left\langle w, \varphi_{i}\right\rangle_{H^{\prime}(D)}=\left(1+\lambda_{i}\right)\left\langle w, \varphi_{i}\right\rangle_{L^{2}(D)} \quad\left(\forall w \in H^{1}(D)\right) .
$$

Thus the functions $\Phi_{i}=\left(1+\lambda_{i}\right)^{-1 / 2} \varphi_{i}$ form an orthonormal basis for $H^{1}(D)$, and the expansion (A1), for $g \in H^{1}(D)$, holds also in $H^{1}(D)$. Moreover, for any $g \in L^{2}(D)$, 
$s_{1}(g)$ represents the formal sum of squares of the Fourier coefficients $\left\langle g, \Phi_{i}\right\rangle_{H^{\prime}(D)}$; it is therefore clear (from standard Hilbert-space theory) that Lemma 1 holds for $k=1$.

Suppose now that $k=2$. Let $Y$ be the space of all finite linear combinations of $\varphi_{i}$, and let $\mathscr{R}^{2}(D)$ denote the closure of $Y$ in $H^{2}(D)$. Then, as $Y \subset N^{2}(D)$ and $N^{2}(D)$ is a closed subspace of $H^{2}(D)$,

$$
\mathscr{R}^{2}(D) \subset N^{2}(D) .
$$

Let $Q$ denote the operator $Q w=-\Delta w+w$. Using standard a priori estimates for the problem

$$
Q w=h \quad \text { in } D, \quad \partial w / \partial n=0 \quad \text { on } \partial D,
$$

we see that the function $\|Q(\cdot)\|_{L^{2}(D)}$ is equivalent to $\|\cdot\|_{H^{2}(D)}$ on $Y$. Let $g \in Y$. Then $g=\sum c_{i} \varphi_{i}$ is a finite sum and

$$
\|Q g\|_{L^{2}(D)}=s_{2}(g) .
$$

Thus (i) and (ii) of Lemma 1 hold with $N^{2}(D)$ replaced by $\mathscr{R}^{2}(D)$. To complete the argument for $k=2$ we must show that

$$
\mathscr{R}^{2}(D)=N^{2}(D)
$$

Since

$$
Q g=\sum\left(1+\lambda_{i}\right) c_{i} \varphi_{i}
$$

for $g \in \mathscr{R}^{2}(D)$, it is clear from (i) of the lemma with $N^{2}(D)$ replaced by $\mathscr{R}^{2}(D)$ that $Q$ maps $\mathscr{R}^{2}(D)$ onto $L^{2}(D)$. On the other hand, the uniqueness theorem for (A4) shows that $Q$ is one-to-one from $N^{2}(D)$ to $L^{2}(D)$. Hence (A5) is valid.

The proof for $k=3$ follows, as above, from the equivalency of $\|\cdot\|_{H^{3}(D)}$ and $\|Q(\cdot)\|_{H^{\prime}(D)}$ on $N^{3}(D)$. (We have such an estimate, since $\partial D$ is $C^{3}$.)

Now let $Q=D \times G\left(D \subset \mathbf{R}^{p}, G \subset \mathbf{R}^{n-p}\right)$ with $\partial D$ and $\partial G$ of class $C^{3}$.

Lemma 2. Let $k$ be a positive integer. Then

$$
L^{2}\left(D ; H^{k}(G)\right) \cap L^{2}\left(G ; H^{k}(D)\right)=H^{k}(\Omega) .
$$

Proof. It suffices to show that if $g$ and $\partial^{k} g / \partial x_{i}^{k}(i=1,2, \ldots, n)$ belong to $L^{2}(\Omega)$, then $g \in H^{k}(\Omega)$. But this follows from the coerciveness theorem of Aronszajn and Smith (cf. [1, Theorem 11.10]).

Let $\left\{\lambda_{i}, \varphi_{i}\right\}$ and $\left\{\alpha_{i}, \psi_{i}\right\}$, respectively, be Neumann systems for $D$ and $G$, and write $\varphi_{i} \psi_{j}$ for the function on $\Omega$ with values $\varphi_{i}(x) \psi_{j}(y), x \in D, y \in G$. Then the collection $\mathscr{B}$ of functions of the form $\varphi_{i} \psi_{j}$ is an orthonormal basis for $L^{2}(\Omega)$, and any function $g \in L^{2}(\Omega)$ admits an expansion of the form

$$
g=\sum_{i, j} c_{i j} \varphi_{i} \psi_{j}
$$

Further, an argument analogous to that used in the proof of Lemma 1 for $k=1$ yields 
LemmA $3 . \mathscr{B}$ is an orthogonal basis for $H^{1}(\Omega)$, and, for $g=\sum_{i, j} c_{i j} \varphi_{i} \psi_{j} \in L^{2}(\Omega)$,

$$
g \in H^{1}(\Omega) \Leftrightarrow \sum_{i, j}\left(1+\lambda_{i}+\alpha_{j}\right) c_{i j}^{2}<\infty .
$$

Proof of the Regularity Lemma. It clearly suffices to show that, given $g \in H^{1}(\Omega)$, if $u \in H^{1}(\Omega)$ satisfies

$$
\langle u, \eta\rangle_{H^{1}(\Omega)}=\langle g, \eta\rangle_{L^{2}(\Omega)} \quad\left(\forall \eta \in H^{1}(\Omega)\right),
$$

then $u \in H^{3}(\Omega)$. (Indeed, replace $g$ in the statement of the Regularity Lemma by $g-u$.) Thus choose $u, g \in H^{1}(\Omega)$ consistent with (A6), and let

$$
u=\sum_{i, j} d_{i j} \varphi_{i} \psi_{j}, \quad g=\sum_{i, j} c_{i j} \varphi_{i} \psi_{j}
$$

The choice $\eta=\varphi_{i} \psi_{j}$ in (A6) yields, with the aid of Green's formula,

$$
c_{i j}=\left(1+\lambda_{i}+\alpha_{j}\right) d_{i j}
$$

On the other hand, since $g \in H^{1}(\Omega)$, Lemma 3 implies that

$$
\sum_{i, j}\left(1+\lambda_{i}+\alpha_{j}\right) c_{i j}^{2}<\infty
$$

hence

$$
\sum_{i, j}\left(1+\lambda_{i}+\alpha_{j}\right)^{3} d_{i j}^{2}<\infty
$$

so that, in particular,

$$
\sum_{i, j}\left(1+\lambda_{i}\right)^{3} d_{i j}^{2}<\infty, \quad \sum_{i, j}\left(1+\alpha_{j}\right)^{3} d_{i j}^{2}<\infty
$$

The first of these inequalities implies that $u \in L^{2}\left(G ; H^{3}(D)\right)$, the second that $u \in$ $L^{2}\left(D ; H^{3}(G)\right)$. Thus, appealing to Lemma $2, u \in H^{3}(\Omega)$.

\section{REFERENCES}

[1] S. Agmon, Lectures on elliptic boundary value problems, Van Nostrand Math. Studies 2, Princeton, 1965

[2] C. Bandle, Isoperimetric inequalities and applications, Pitman, Boston, 1980

[3] J. W. Cahn, On spinodal decomposition, Acta Met. 9, 795-801 (1961)

[4] J. W. Cahn and J. E. Hilliard, Free energy of a nonuniform system. I. Interfacial free energy, J. Chem. Phys. 28, 258-267 (1958)

[5] J. W. Cahn and J. E. Hilliard, Spinodal decomposition: a reprise, Acta Met. 19, 151-161 (1971)

[6] A. P. Calderon, Uniqueness in the Cauchy problem for partial differential equations, Amer. J. Math. 80, 16-36 (1958)

[7] J. Carr, M. E. Gurtin, and M. Slemrod, Structured phase transitions on a finite interval, Arch. Rat. Mech. Anal. 86, 317-351 (1984)

[8] R. G. Casten and C. J. Holland, Instability results for reaction diffusion equations with Neumann boundary conditions, J. Differential Equations 27, 266-273 (1978)

[9] N. Chafee, Asymptotic behavior for solutions of a one-dimensional parabolic equation with homogeneous Neumann boundary conditions, J. Differential Equations 18, 111-134 (1975)

[10] E. Conway, D. Hoff, and J. Smoller, Large time behavior of systems of nonlinear reaction-diffusion equations, SIAM J. Appl. Math. 35, 1-16 (1978)

[11] P. Grisvard, Elliptic problems in nonsmooth domains, Pitman, Boston, 1984 
[12] P. Hartman and A. Wintner, On the local behavior of solutions of non-parabolic partial differential equations, Amer. J. Math. 75, 449-476 (1953)

[13] S. Jimbo, Singular perturbations of domains and semilinear elliptic equations, J. Fac. Sci. Univ. Tokyo. To appear.

[14] B. Kawohl, Rearrangements and convexity of level sets in PDE, Lecture Notes in Math. 1150, SpringerVerlag, Berlin, 1985

[15] B. Kawohl, On the isoperimetric nature of a rearrangement inequality and its consequences for some variational problems, Arch. Rat. Mech. Anal. 94, 227-243 (1986)

[16] H. Matano, Asymptotic behavior and stability of solutions of semilinear diffusion equations, Publ. Res. Inst. Math. Sci. 15, 401-454 (1979)

[17] H. Matano, Nonincrease of the lap-number of a solution for a one-dimensional parabolic equation, $\mathbf{J}$. Fac. Sci. Univ. Tokyo Sec. IA 29, 401-441 (1982)

[18] L. E. Payne and H. F. Weinberger, An optimal Poincaré inequality for convex domains, Arch. Rat. Mech. Anal. 5, 286-292 (1960)

[19] J. S. Rowlinson, Translation of J. D. van der Waals' "The thermodynamic theory of capillarity under the hypothesis of a continuous variation of density," J. Statist. Phys. 20, 197-244 (1979)

[20] J. D. van der Waals, The thermodynamic theory of capillarity under the hypothesis of a continuous variation of density (in Dutch), Verh. Konink. Akad. Wetensch. Amsterdam (Sect. 1) Vol. 1, No. 8 (1893) 